\title{
Computer-assisted TKA: long-term outcomes at a minimum follow-up of 10 years of 129 e- motion FP mobile bearing prostheses.
}

\author{
Dominique Saragaglia ${ }^{1}$, Olivier Seurat ${ }^{1}$, Régis Pailhé ${ }^{1}$, Brice Rubens Duval ${ }^{1}$ \\ ${ }^{1}$ Grenoble South Teaching Hospital, Échirolles, 38130, France.
}

\begin{abstract}
The hypothesis was that long-term outcomes were satisfactory or superior to the other implants design reported outcomes. This study confirms our initial hypothesis, that the outcomes of the TKA e-Motion FP with ultra-congruent mobile-bearing were satisfactory at more than 10 years of follow-up. Navigation, whose accuracy is well established, probably contributed to the quality of the results.
\end{abstract}

\section{Introduction}

Mobile bearing TKA is currently one of the best mean to deal with knee osteoarthritis $(1,2)$. Computer assisted knee replacement is used since more than 15 years but long term results of this technology are not well known. The aim of this study was to assess long-term outcomes of the e-Motion FP ultra congruent TKA with mobile-bearing platform, setup with computer assisted surgery. Our null hypothesis was that long-term outcomes were satisfactory (NICE criteria) or superior to the other implants design reported outcomes.

\section{Material and methods}

We conducted a retrospective monocentric study between January 2002 and December 2005, on 243 knees in 225 patients ( 22 bilateral) of a mean age of $71+/-5$ years (65-85) who underwent a primary total knee arthroplasty. The series consists of 222 knee osteoarthritis, 7 rheumatoid arthritis and 14 osteonecrosis of the femoral condyles. 13 patients were operated previously: 7 for medial or lateral meniscectomy, 3 for an HTO, 1 for a fracture of the distal femur, 1 for an ACL reconstruction and the last for a patellar osteoarthritis. 147 knees were in varus $\left(\mathrm{HKA}<177^{\circ}\right), 44$ in neutral alignment $\left(\mathrm{HKA}\right.$ between $177^{\circ}$ and $183^{\circ}$ ) and 42 were in valgus $\left(\mathrm{HKA}>183^{\circ}\right)$. The mean preoperative HKA angle was $170.6^{\circ} \pm 6.3^{\circ}\left(160-176^{\circ}\right)$ for the genu varum deformities, $188.5^{\circ} \pm 4.1^{\circ}\left(184-200^{\circ}\right)$ for genu valgum and $179.5^{\circ} \pm 3.5^{\circ}\left(177-183^{\circ}\right)$ for neutral alignments. 
Computer-Assisted TKA : Long-Term Outcomes at a Minimum Follow-Up of .... D. Saragaglia et al.

The mean preoperative International Knee Society (IKS) score was $82.1 \pm 25.1$ point (14-158) with a knee score of $36.1 \pm 16.3$ points (10-87) and a function score of $44.7 \pm 14.8$ points $(0$ 90). The mean preoperative flexion angle was $121 \pm 11^{\circ}\left(90-140^{\circ}\right)$. The e-Motion ${ }^{\mathrm{TM}}$ TKA (BBraun-Aesculap, Tuttlingen, Germany) is a fully congruent mobile bearing prosthesis from 0 to $60^{\circ}$ of flexion with a floating platform. All prostheses were navigated with Orthopilot ${ }^{\mathrm{TM}}$ which is a non-image-based navigation device.

\section{Results}

129 knees in 117 patients were reviewed at a mean follow-up of $135 \pm 12.8$ months (120-165). 108 patients were lost to follow-up among which 76 were dead. Only one aseptic loosening was found at 10 years of follow-up. No complication concerning wear or tibial plateau insert dislocation was noted. 3 postoperative infections were noted, 1 after 5 months of follow up, 1 after one year and the last after 6 years. Only one case needed revision of the prosthesis because of loosening of the tibial implant. The mean prosthesis survival rate at 11.3 years was $99.2 \%$ with aseptic loosening, wear or failure of the prosthesis as an endpoint. It was $96.9 \%$, if we included revisions due to septic loosening. The mean IKS score at the last follow-up was 189.5 \pm 13.6 points $(137-200)$ with IKS knee score of $96 \pm 6$ points $(70-100)$ and a function score of $91 \pm 10$ points $(55-100)$. The mean angle flexion was $119^{\circ}+/-12^{\circ}\left(80^{\circ}-140^{\circ}\right)$.

The mean HKA angle was $180 \pm 2^{\circ}\left(174-186^{\circ}\right)$ with $92.3 \%$ of the prostheses aligned at 180 $\circ+/-3^{\circ}$ which was the preoperative objective.

\section{Discussion-conclusion}

This study confirms our initial hypothesis, that the outcomes of the TKA e-Motion FP with ultra-congruent mobile-bearing were satisfactory at more than 10 years of follow-up. These results are comparable to other series of the literature at the same follow-up (3). Navigation, whose accuracy is well established $(4,5)$, probably contributed to the quality of the results. Very Long-term outcomes need to be further investigated to demonstrate a superiority or not of mobile bearing devices.

\section{References}

1- Buechel FF (2002). Long-term follow-up after mobile-bearing total knee replacement. Clin Orthop, 404, pp: 40-50.

2- Kim Y-H, Yoon S-H, Kim J-S (2007). The long-term results of simultaneous fixed-bearing and mobile-bearing total knee replacements performed in the same patient. Bone Joint J, 89B, pp:1317-23.

3- Argenson J-N, Parratte S, Ashour A, Saintmard B, Aubaniac J-M (2012). The Outcome of Rotating-Platform Total Knee Arthroplasty with Cement at a Minimum of Ten Years of Follow-up. J Bone Jt Surg, 94(7), pp: 638-44.

4- Hoffart H-E, Langenstein E, Vasak N (2012). A prospective study comparing the functional outcome of computer-assisted and conventional total knee replacement. J Bone Joint Surg, 94-B, pp:194-9. 
Computer-Assisted TKA : Long-Term Outcomes at a Minimum Follow-Up of .... D. Saragaglia et al.

5- Blakeney WG, Khan RJK, Palmer JL (2014). Functional outcomes following total knee arthroplasty: a randomised trial comparing computer-assisted surgery with conventional techniques. The Knee, 21(2), pp :364-8.

D. Saragaglia receives royalties from BBraun-Aesculap for the e-Motion prosthesis. 